\title{
Chitosan as Flocculant Agent for Clarification of Stevia Extract
}

\author{
Silvia P. D. de Oliveira, Cynthia R. A. Mahl, Márcia R. Simões \\ Centro de Engenharias e Ciências Exatas, UNIOESTE \\ Classius F. da Silva \\ Centro de Ciências Exatas e da Terra, UNIFESP
}

\begin{abstract}
Stevia is used as a sweetener due to its low calorific value and its taste, which is very similar to that of sucrose. After extraction from dried leaves, stevia extract is dark in colour, and therefore needs to be whitened to increase acceptance by consumers. In this study we tested chitosan, a cationic polyelectrolyte, as flocculant agent for the whitening of the Stevia extract. Positive charges of chitosan can interact electrostatically with a counter-ion, sodium tripolyphosphate (TPP), and then chitosan precipitates. A factorial design was used to study the whitening process, in which Glycosides Removal, Colour Removal, Turbidity Removal and Soluble Solids Removal were evaluated. The studied factors were Chitosan Mass and $\mathrm{pH}$ of the TPP solution. The results showed that chitosan is a good flocculant agent, being able to flocculate both the glycosides and the pigments that make the extract coloured.
\end{abstract}

Keywords: Stevia, sweeteners, chitosan, flocculation, clarification.

\section{Introduction}

The leaves of the sweet herb from Paraguay, Stevia rebaudiana Bertoni, produce an alternative to sucrose with the advantage that stevia sweeteners are natural plant products, non-metabolizable, non-fermentable, anti-carcinogenic, anti-plaque and non-toxic to humans. In addition, the functional and sensory properties of stevia are superior to those of many other high-potency sweeteners ${ }^{[1]}$. Global markets for Stevia is pegged at around \$ 500 million with Japan alone accounting for $\$ 200$ million $^{[2]}$.

According to Crammer and $\mathrm{Ikan}^{[3]}$, the leaves of the Stevia rebaudiana plant contain a complex mixture of diterpenes, triterpenes, tannins, stigmasterol, volatile oils and eight sweet diterpene glycosides. They are Stevioside, Steviolbioside, Rebaudiosides A, B, C, D and E and Dulcoside A (Figure 1). Rebaudioside $\mathrm{A}$ is more stable and much sweeter and tastes better than Stevioside. Rebaudioside E is as sweet as Stevioside, and Rebaudioside D is as sweet as Rebaudioside A, while the remaining diterpene glycosides are not as sweet as Stevioside. Although there is a molecule called Stevioside, it is customary to employ the term Steviosides to the set of sweet compounds.

The relative sweetness of these diterpenes glycosides depends on concentration and the environment. Collectively they make stevia 100 to 300 times sweeter than sucrose ${ }^{[4]}$.

Dried leaves are infused in hot water or alcohol (ethanol or methanol) to obtain their extract, which is a solution with brownish colloidal particles. This extract must be clarified prior to commercialization. One method of clarification is precipitation of impurities by hydroxides and fractional extraction of glycosides with butanol or methanol ${ }^{[5,6]}$. These solvents are usually toxic to humans and after clarification they have to be totally removed from the sweetener. Others processes have been used in the clarification, like filtration ${ }^{[7,8]}$, flocculation with polyelectrolytes ${ }^{[9,10]}$ and adsorption $^{[11,12]}$.

Flocculation appears as a promising process since it is safe and free of toxic solvents. Bunhak et al. ${ }^{[10]}$ investigated two polyelectrolytes on the clarification of Stevia extract using two commercial flocculants available in Brazil, NOVUS ${ }^{\mathrm{TM}}$ CE2666 (cationic polyelectrolyte) and BETZDEARBORN ${ }^{\mathrm{TM}}$ F11 (anionic polyelectrolyte). The best result was obtained when the anionic polyelectrolyte was used, achieving a turbidity removal of equivalent to $97 \%$. Furthermore, Fernandes et al. ${ }^{[9]}$ tested the cactus Cereus peruvianus as an alternative natural resin to clarify the stevia extract. This cactus behaved like a strong acid resin and eliminated about $95 \%$ of the colour and turbidity of stevia.

Natural polymers, such as chitosan, have advantages because they are cheaper and safer from the point of view of food. The main properties of this polysaccharide are: bioactivity, biodegradability, biocompatibility, reactivity of deacetylated amino groups, selective permeability, polyelectrolyte action, ability to form gel film, chelation ability and adsorption capacity $^{[13,14]}$.

Chitosan has unique properties among biopolymers, especially due to the presence of primary amino groups. Chitosan has been used for the chelation of metal ions in near-neutral solution, the complexation of anions in acidic solution (cationic properties due to amine protonation), the coagulation of negatively charged contaminants under acidic conditions, and for precipitative flocculation at $\mathrm{pH}$ above the $\mathrm{pKa}$ of chitosan. The coagulation and flocculation properties can be used to treat particulate suspensions (organic or inorganic) and also to treat dissolved organic materials (including dyes and humic acid). Moreover, chitosan has characteristics of both coagulants and flocculants agents: high cationic charge density, long polymer chains, bridging of aggregates, and precipitation in neutral or alkaline $\mathrm{pH}$ conditions ${ }^{[15]}$. These properties have been exploited for the design of coagulation-flocculation processes applied, for example, to sewage treatment ${ }^{[16]}$, industry wastewater ${ }^{[17]}$, piggery wastewater treatment ${ }^{[18]}$, paper-recycling wastewater ${ }^{[19]}$.

According to Guibal et al. ${ }^{[15]}$, stoichiometry and charge restabilization were determined for the coagulation of humic acid solutions, kaolin suspensions, and organic dyes with chitosan, indicating charge neutralization as the dominant mechanism for removal. Charge patch destabilization and bridging mechanisms were inferred in organic suspensions, based on the effects of the apparent molar mass of the chitosan preparations and effectiveness of sub-stoichiometric doses of chitosan.

This work aims to study the clarification of aqueous stevia extract in a batch reactor using chitosan as flocculant agent. Factorial design was used to study the process and the studied factors were: $\mathrm{pH}$ of the sodium tripolyphosphate solution and the Chitosan Mass. 


\section{Material and Methods}

\section{Material}

Stevia leaves were kindly supplied by Steviafarma S.A. (Maringá, Brazil). Chitosan (viscosimetric molar mass $=105 \mathrm{kDa}$; degree of deacetylation $=81.61 \pm 1.05 \%$ ) was purchased from Polymar (Fortaleza, Brazil). All other chemicals (phenol, sulfuric acid, sodium tripolyphosphate) were of analytical grade used without further purification. The solutions were prepared using distilled water.

\section{Preparation of chitosan solution}

The chitosan solution, with a concentration of $0.66 \% \mathrm{w} / \mathrm{w}$, was obtained by dissolving chitosan powder in 0.25 vol. \% acetic acid solution and magnetic stirring for at least 24 hours.

\section{Preparation of aqueous stevia extract}

The extract was prepared by mixing one part of stevia leaves with nine parts of water. For such purpose, water was heated to $55^{\circ} \mathrm{C}$ and then mixed with stevia leaves, with the mixture being allowed to steep for 1 hour. After cooling, the extract was filtered through a paper filter and centrifuged at $3000 \mathrm{rpm}$ for 15 minutes.

\section{Clarification experiments}

Ten milliliters of Stevia extract was added in $5 \mathrm{~mL}(3.3 \mathrm{~g}$ of chitosan) or $10 \mathrm{~mL}$ ( $6.6 \mathrm{~g}$ of chitosan) of chitosan solution and kept under magnetic stirring at room temperature for 15 minutes. Then ten milliliters of sodium tripolyphosphate (TPP) solution $(1 \% \mathrm{w} / \mathrm{v})$ was added and stirred for 15 minutes. The $\mathrm{pH}$ of TPP solutions was adjusted to the required value by the addition of concentrated $\mathrm{HCl}$. The suspension was left to decanter for 15 minutes and then

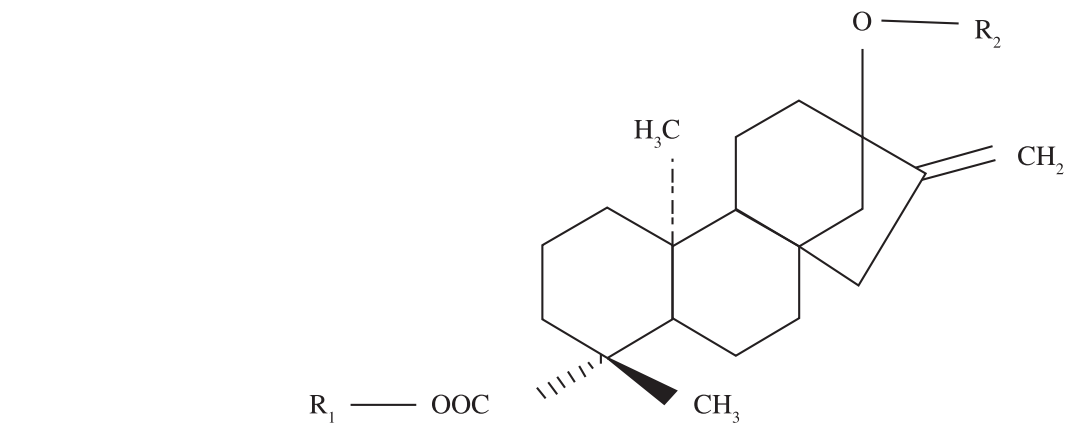

\begin{tabular}{|c|c|c|c|}
\hline Diterpenes glycosides & $\mathrm{R}_{1}^{\mathrm{a}}$ & $\mathrm{R}_{2}{ }^{\mathrm{a}}$ & $\begin{array}{c}\text { Sweetner } \\
\text { potency } \\
(\text { sucrose = 1) }\end{array}$ \\
\hline Steviolbioside & $\mathrm{H}$ & $\mathrm{glc}^{2}-{ }^{1} \mathrm{glc}$ & $100-125$ \\
\hline Rubusosídeo & glc & glc & $100-120$ \\
\hline Stevioside & $\mathrm{glc}$ & $\mathrm{glc}^{2}-{ }^{1} \mathrm{glc}$ & $150-300$ \\
\hline Rebaudioside A & glc & $\mathrm{glc}_{3}{ }^{2}{ }^{1} \mathrm{glc}$ & $250-450$ \\
\hline Rebaudioside B & $\mathrm{H}$ & & $300-350$ \\
\hline $\begin{array}{c}\text { Rebaudioside C } \\
\text { (dulcoside B) }\end{array}$ & glc & glc & $120-500$ \\
\hline Rebaudioside D & $\mathrm{glc}^{2}-{ }^{1} \mathrm{glc}$ & $\mathrm{glc}_{3}{ }^{2}-{ }^{1} \mathrm{glc}$ & $250-450$ \\
\hline Rebaudioside E & $\mathrm{glc}^{2}{ }^{1} \mathrm{glc}$ & $\mathrm{glc}^{2}-{ }^{1} \mathrm{glc}$ & $150-300$ \\
\hline Dulcoside A & glc & $\mathrm{glc}^{2}{ }^{1}$ rham & $50-120$ \\
\hline
\end{tabular}

${ }^{a}$ glc, $\beta$-D-glucopyranosyl; rham, $\alpha$-L-ramnopyranosyl.

Figure 1. Structure of some stevia glycosides ${ }^{[3]}$. 
was centrifuged at $3000 \mathrm{rpm}$ for 15 minutes and the supernatant was analyzed. A $2^{2}$ factorial design without central points was performed to evaluate the importance of the $\mathrm{pH}$ of TPP solution and Chitosan Mass on the clarification process of stevia extract. Table 1 summarizes these factor and their respective levels. The $\mathrm{pH}$ and the polyelectrolyte mass (Chitosan Mass) are important factors in flocculation processes with polyelectrolytes. Our preliminary studies, also using the response surface methodology showed that, specifically in the case studied, the concentration of chitosan had no significant effect. Thus, the levels chose in this factorial design were also determined from our previous experience. All the essays were performed in triplicate and according to the Table 2.

Parameters such as the Colour Removal (CR), Turbidity Removal (TR), Total Glycosides Removal (GR) and Soluble Solids Removal (SR) were evaluated employing Equation 1:

$$
\text { Parameter }=\left(\frac{\text { property }_{\text {BEFORE }}-\text { property }_{A F T E R}}{\text { property }_{B E F O R E}}\right) \times 100(\%)
$$

The subscripts "BEFORE" and "AFTER" relate to the properties before and after the clarification process, respectively. Properties like optical absorbancies measured at 420 and $670 \mathrm{~nm}$ were correlated with the Colour Removal (CR) and Turbidity Removal (TR) parameters, respectively ${ }^{[20]}$. Each parameter was calculated with respect to a solution having the same $\mathrm{pH}$ value.

According to Costa et al. ${ }^{[21]}$, it is possible to relate the concentration of total stevia glycosides with the concentration of total carbohydrate which can be obtained via the phenol/sulphuric acid method. The quantities of free sugars or sugar oligomers present in the extract are insignificant. The adsorption percentage of stevia glycosides was also calculated by Equation 1 using the concentration of total stevia glycosides as a property. Absorbancies were analyzed employing a spectrophotometer (Shimadzu UV

Table 1. Factors and levels used in the $2^{2}$ factorial design study.

\begin{tabular}{lll}
\hline \multicolumn{1}{c}{ Factors } & \multicolumn{2}{c}{ Levels } \\
\cline { 2 - 3 } & $\mathbf{( - 1 )}$ & $\mathbf{( + 1 )}$ \\
\hline pH of TPP solution & 7.5 & 9.3 \\
Chitosan Mass, CM $(\mathrm{g})$ & $3.3 *$ & $6.6 * *$ \\
\hline *Chitosan Mass contained in $5 \mathrm{~mL}$ of chitosan solution $(0.66 \% \mathrm{w} / \mathrm{w}) ;$ \\
$* *$ Chitosan Mass contained in $10 \mathrm{~mL}$ of chitosan solution $(0.66 \% \mathrm{w} / \mathrm{w})$.
\end{tabular}

1601 PC, Japan). The total soluble solids in a given solution were determined by refractometer (Atago, Japan). This enabled the soluble solids properties, called ${ }^{\circ}$ Brix, to be obtained. The total percentage adsorption of soluble solids was calculated via Equation 1 using the measured ${ }^{\circ}$ Brix as a given property.

\section{Statistical analysis}

The statistical calculations were performed using the Statistica ${ }^{\circledR}$ software packages.

\section{Results and Discussion}

Table 2 shows the experimental conditions used in this study with the results for Glycosides Removal (GR), Colour Removal (CR), Turbidity Removal (TR), and Soluble Solids Removal (SR).

The effects estimates for each variable were determined and reported in Table 3. An estimate effect is obtained by evaluating the difference in process performance caused by a change from the low $(-1)$ to the high $(+1)$ level of the corresponding factor ${ }^{[22]}$. A small $p$-value suggested that the coefficient was a large signal in comparison to the noise because it was too large to have arisen by chance alone. In this case, $p \leq 0.05$, suggested significance at the 0.05 level. This also corresponded to a $95 \%$ confidence level for a test of the hypothesis that the effects in question were equal to zero.

The $\mathrm{R}$-squared $\left(\mathrm{R}^{2}\right)$ value provides a measure of how much of the variability in the observed response values could be explained by the experimental factors and their interactions. Models with R-square values above 0.9 are considered excellent and can explain most of the variation in the response. Actually, models with R-squared near the unity present better response predictions, minimizing the difference between the predicted and observed values ${ }^{[22]}$. According to this statement, only the GR presented an adequate predicting model. Every parameter is discussed separately as following.

\section{Glycosides Removal (GR)}

Table 3 shows the effect of the factors on the percentage of Glycosides Removal (GR). The average effect was about $44.56 \%$, which is not acceptable since stevia glycosides should not be flocculated by the flocculant agent. Our purpose is only to clarify the extract, so it is important to minimize the flocculation of stevia glycosides, i.e., the

Table 2. Actual values and coded levels (in parentheses) for the factorial design and the results for Glycosides Removal (GR), Colour Removal (CR), Turbidity removal (TR), and Soluble Solids Removal (SR).

\begin{tabular}{|c|c|c|c|c|c|c|}
\hline Experiment & $\mathrm{CM}(\mathrm{g})^{*}$ & $\mathbf{p H}^{* * *}$ & GR (\%) & CR (\%) & TR (\%) & SR $(\%)$ \\
\hline \multirow{4}{*}{1} & \multirow{3}{*}{$3.3(-1)$} & \multirow{3}{*}{$7.5(-1)$} & 63.83 & 92.86 & 88.89 & 55.56 \\
\hline & & & 63.25 & 94.64 & 88.89 & 55.56 \\
\hline & & & 51.80 & 91.07 & 77.78 & 44.44 \\
\hline & \multicolumn{2}{|c|}{ Mean $\pm S D^{* * *}$} & $59.63 \pm 6.78$ & $92.86 \pm 1.79$ & $85.19 \pm 6.41$ & $51.85 \pm 6.42$ \\
\hline \multirow{4}{*}{2} & \multirow{3}{*}{$3.3(-1)$} & \multirow{3}{*}{$9.3(+1)$} & 23.83 & 85.19 & 89.47 & 50.00 \\
\hline & & & 29.69 & 68.89 & 84.21 & 40.00 \\
\hline & & & 29.90 & 81.48 & 100.00 & 50.00 \\
\hline & \multicolumn{2}{|c|}{ Mean $\pm S D$} & $27.81 \pm 3.45$ & $78.52 \pm 8.54$ & $91.23 \pm 8.04$ & $46.67 \pm 5.77$ \\
\hline \multirow{4}{*}{3} & \multirow{3}{*}{$6.6(+1)$} & \multirow{3}{*}{$7.5(-1)$} & 42.28 & 88.83 & 69.23 & 66.67 \\
\hline & & & 43.56 & 93.85 & 96.15 & 66.67 \\
\hline & & & 36.25 & 90.50 & 69.23 & 55.56 \\
\hline & \multicolumn{2}{|c|}{ Mean $\pm S D$} & $40.70 \pm 3.90$ & $91.06 \pm 2.56$ & $78.20 \pm 15.54$ & $62.97 \pm 6.41$ \\
\hline \multirow{4}{*}{4} & \multirow{3}{*}{$6.6(+1)$} & \multirow{3}{*}{$9.3(+1)$} & 49.90 & 87.25 & 100.00 & 66.67 \\
\hline & & & 48.12 & 85.23 & 90.48 & 55.56 \\
\hline & & & 52.35 & 87.25 & 95.24 & 55.56 \\
\hline & Average $\pm S D$ & & $50.12 \pm 2.12$ & $86.58 \pm 1.17$ & $95.24 \pm 4.76$ & $59.26 \pm 6.41$ \\
\hline
\end{tabular}

*Chitosan Mass $(\mathrm{CM}),{ }^{*} \mathrm{pH}$ of TPP Solution, ***SD: Standard Deviation. 
flocculant agent must be able to flocculate substances that give colour to the extract without flocculating stevia glycosides.

The $\mathrm{pH}$ showed negative effect on the percentage of the GR what is desirable as it must be minimized. However the effect of $\mathrm{pH}$ can not be analyzed alone, since the interaction between Chitosan Mass and $\mathrm{pH}$ was significant $(\mathrm{p} \leq 0.05)$. Figure 2 shows the interactions between the $\mathrm{pH}$ and Chitosan Mass. The Vertical bars in the Figure 2 denote 0.95 confidence intervals.

According to the Figure 2, at the $\mathrm{pH}=7.5$ (the lower level, i.e., -1 ), the highest level of Chitosan Mass (6.6 g) presented the lowest GR values. On the other hand, at $\mathrm{pH}=9.3$ (the higher level, i.e., +1 ), the highest level of Chitosan Mass (6.6 g) presented the highest GR values. So, there is a contrary behavior, which characterizes the interaction between Chitosan Mass and $\mathrm{pH}$. This behavior can also be seen in the response surface presented in Figure 3. As expected, the lowest GR value occurs on the lower Chitosan Mass and higher $\mathrm{pH}$.

Guibal et al. ${ }^{[15]}$ studied the coagulation of an anionic dye whit chitosan. For a given dye concentration, increasing the chitosan

Table 3. Effect values and their standard errors for Glycosides Removal (GR), Colour Removal (CR), Turbidity Removal (TR), and Soluble Solids Removal (SR) at 95\% confidence level.

\begin{tabular}{crrcc}
\hline Effect & \multicolumn{1}{c}{ GR } & CR & TR & SR \\
\hline Average & $\mathbf{4 4 . 5 6} \pm \mathbf{1 . 2 7}$ & $87.25 \pm 1.32$ & $\mathbf{8 7 . 4 6} \pm \mathbf{2 . 7 8}$ & $\mathbf{5 5 . 1 9} \pm \mathbf{1 . 8 1}$ \\
Main & & & & \\
V & $1.69 \pm 2.54$ & $3.13 \pm 2.65$ & $-1.48 \pm 5.55$ & $\mathbf{1 1 . 8 6} \pm \mathbf{3 . 6 2}$ \\
pH & $\mathbf{- 1 1 . 2 0} \pm \mathbf{2 . 5 4}$ & $\mathbf{- 9 . 4 1} \pm \mathbf{2 . 6 5}$ & $11.54 \pm 5.55$ & $-4.44 \pm 3.62$ \\
Interactions & & & & \\
$\quad$ V $\times$ pH & $\mathbf{2 0 . 6 2} \pm \mathbf{2 . 5 4}$ & $4.93 \pm 2.65$ & $5.50 \pm 5.55$ & $0.74 \pm 3.62$ \\
\hline
\end{tabular}

*Significant factors in bold letters $(\mathrm{p} \leq 0.05)$; $\mathrm{GR}\left(\mathrm{R}^{2}=0.9158\right) ; \mathrm{CR}$ $\left(\mathrm{R}^{2}=0.6863\right) ; \mathrm{TR}\left(\mathrm{R}^{2}=0.4000\right) ; \mathrm{SR}\left(\mathrm{R}^{2}=0.6100\right)$

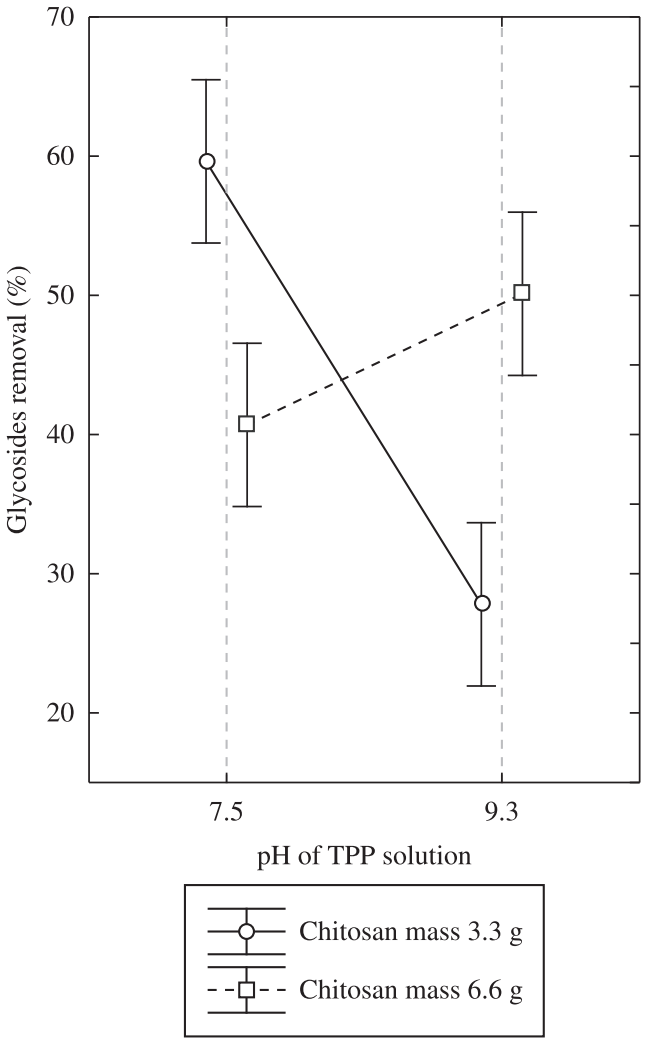

Figure 2. Interactions between the Chitosan Mass and $\mathrm{pH}$ for the Glycosides Removal ( $\mathrm{p} \leq 0.05)$. concentration decreased the residual concentration of the dye up to an upper limit of chitosan concentration. Above this limit concentration (optimum chitosan concentration, OCC) the addition of an excess of chitosan resulted in a re-stabilization of the dye and a decrease in coagulation efficiency. This optimum chitosan dosage (OCC) strongly depended on both the initial $\mathrm{pH}$ and the dye concentration. The OCC provided a precise measurement of the effective chitosan concentration, establishing the stoichiometric ratio between the functional groups of the dye and the biopolymer. Therefore, the OCC could explain the interaction between the $\mathrm{pH}$ and Chitosan Mass. At one of the $\mathrm{pH}$, the Chitosan Mass is below the OCC, and at the other $\mathrm{pH}$, the Chitosan Mass is above the OCC.

\section{Colour Removal (CR)}

The CR values (Table 2) were higher than the one obtained by Bunhak et al. ${ }^{[10]}$ using anionic or cationic polyelectrolytes in order to clarify the stevia extract. The highest Colour Removal verified by Bunhak et al. ${ }^{[10]}$ was $79.2 \%$ and $72.2 \%$ for anionic and cationic polyelectrolytes, respectively. Moreover, the average effect was about $87.25 \%$ (Table 3 ) and only the $\mathrm{pH}$ presented significant effects. The negative effect of $\mathrm{pH}$ was also verified by Bunhak et al. ${ }^{[10]}$ with the cationic synthetic polyelectrolyte (NOVUS $\left.{ }^{\mathrm{TM}} \mathrm{CE} 2666\right)$ for the $\mathrm{pH}$ ranging between 8 and 9. On the other hand, they found positive effect on the Colour Removal when the $\mathrm{pH}$ ranged between 9 and 10 .

Fernandes et al. ${ }^{[9]}$ studied the cactus Cereus peruvianus as an alternative natural resin to clarify the original stevia extract. This cactus behaves as a strong acid resin and reached about $95 \%$ of $\mathrm{CR}$ and the $\mathrm{pH}$ was kept bellow 3 .

\section{Turbidity Removal (TR)}

None of the variables (Chitosan Mass and $\mathrm{pH}$ ) had significant effect, even the interaction between them. Only the average effect was significant $(p \leq 0.05)$. The average effect was about $87 \%$ that is very close to the $84 \%$ which was obtained by Fernandes et al. ${ }^{[9]}$. However, they used concentration of resin from 2 to $10 \% \mathrm{w} / \mathrm{v}$, and our results were obtained with just $0.66 \%$ w/v chitosan solution.

\section{Soluble Solids Removal (SR)}

Concerning the SR, the average effect was about $55.19 \%$ (Table 3). The Chitosan Mass was the only significant variable for the SR $(p \leq 0.05)$, so SR increases about $11.86 \%$ when Chitosan Mass increases from $3.3(-1)$ to $6.6 \mathrm{~g}(+1)$.

The soluble solids content observed in stevia extract were from 1.5 to $3.0^{\circ}$ Brix after clarification with chitosan as flocculant agent. Fernandes et al. ${ }^{[9]}$ found similar values (2.0 and $2.7^{\circ}$ Brix) in their study of the cactus Cereus peruvianus on colour removal of the stevia extract.

Mahl et al. ${ }^{[12]}$ tested alginate beads as adsorbent in clarification of stevia extract. They state that an ideal adsorbent for the clarification process must be capable of removing both colour and turbidity, but not removing the glycosides. The clarification was evaluated by determining the ratio of colour removal to glycosides removal. High ratios are associated to process with good performance. This ratio was also used to evaluate the performance of the experiments carried out in this work as shown in Table 4. Based on CR values it is clear that all the experiments lead to good results. Although the experiment 2 had the lowest percentage of $\mathrm{CR}$, such experiment showed the lowest GR. So, it was considered the best conditions to achieve the objectives of this study. The best results were obtained at a $\mathrm{pH}$ value of 9.3 and Chitosan Mass of $3.3 \mathrm{~g}$. The values of the CR/GR ratio were very similar to those obtained by Mahl et al. ${ }^{[12]}$ 


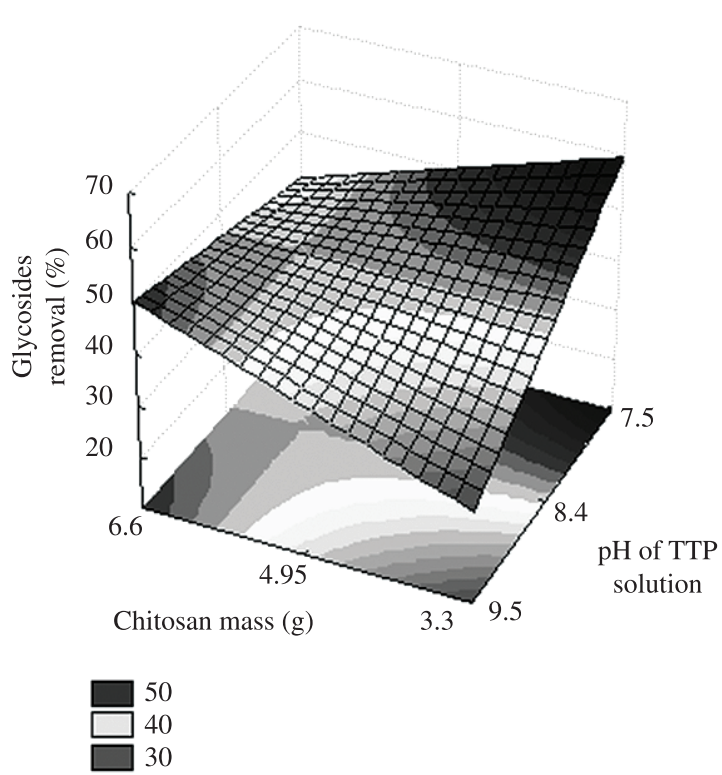

Figure 3. Glycosides Removal (GR) as function of $\mathrm{pH}$ and Chitosan Mass: response surface.

Table 4. Ratio of the Colour Removal (CR) to the Glycosides Removal (GR) for the experiments.

\begin{tabular}{cccc}
\hline Experiment & $\mathbf{C M}(\mathbf{g}) *$ & $\mathbf{p H} * *$ & $\mathbf{C R} / \mathbf{G R}$ \\
\hline 1 & $3.3(-1)$ & $7,5(-1)$ & $1,57 \pm 0,16$ \\
2 & $3.3(-1)$ & $9,3(+1)$ & $2,87 \pm 0,64$ \\
3 & $6.6(+1)$ & $7,5(-1)$ & $2,25 \pm 0,21$ \\
4 & $6.6(+1)$ & $9,3(+1)$ & $1,73 \pm 0,05$ \\
\hline
\end{tabular}

*Chitosan Mass, **pH of TPP Solution.

However, this ratio can not be evaluated alone, because it is not observed CR values greater than $70 \%$.

The experiment 1 would be considered the best results since the CR was highest, but the GR was also high. For the experiment 3, at the same $\mathrm{pH}$ 7.5, when the Chitosan Mass increases from 3.3 to $6.6 \mathrm{~g}$, the GR decreases. A possible explanation for this behavior can be associated to chitosan molecules interactions with both glycosides and TPP. It is also probable that there is a competition between each other (glycosides and TPP).

According to Renault et al. ${ }^{[23]}$, the mechanisms of coagulation/ flocculation involved in the removal of dissolved and particulate contaminants using chitosan often cited are charge neutralization, adsorption (related to protonated amine groups), precipitative coagulation, bridge formation (related to the high molar mass of biomacromolecules) and electrostatic patch. The mechanism is the following: coagulation by charge neutralization destabilizes colloidal impurities and transfers small particles into large aggregates (bridge formation) and adsorbs dissolved organic substances onto the aggregates by an adsorption mechanism which can then be removed easily by filtration and sedimentation.

In the clarification of stevia extract with chitosan, after the addition of chitosan to the stevia extract, it was not observed turbidity of the medium, nor the formation of any particles, indicating that there is no electrostatic interaction between chitosan and stevia extract compounds (no charge neutralization). It is well established ionic crosslinking of chitosan with TPP in order to produce chitosan nanoparticles. On the other hand, increasing the $\mathrm{pH}$ of the medium also may promote the precipitation of chitosan. The removal of some pigments and steviosides from extract occurred during the formation of these nanoparticles and their subsequent aggregation and precipitation. Then, the results suggest that the precipitative coagulation is the most relevant mechanism in the clarification of stevia extract, but the bridging formation must also be occurring.

\section{Conclusions}

Chitosan acted as one good flocculant agent in the clarification of Stevia extract. The Colour Removal (CR) values were from 78 to $93 \%$, which is very good for clarification process of stevia extract. One response surface was also obtained for Glycosides Removal (GR) as function of $\mathrm{pH}$ and Chitosan Mass. The $\mathrm{pH}$ showed a significant effect on the Colour Removal (CR) and Glycosides Removal (GR). While the Chitosan Mass affected only the Soluble Solids Removal (SR). The Turbidity Removal (TR) was not affected by the two variables. Thus, according to the CR/GR ratio, the best results were observed for experiment 2, in which the Chitosan Mass is equal to $3.3 \mathrm{~g}$ and $\mathrm{pH}$ of TPP is equal to 9.3. Moreover, this experiment also is more economic considering the chitosan use. The use of a biodegradable polymer from a renewable resource and less aggressive for the environment is an important criterion for future developments.

\section{References}

1. Brandle, J. E.; Starratt, A. N. \& Gijzen, M. - Can. J. Plant Sci., 78, p.527 (1998). http://dx.doi.org/10.4141/cjps 10086

2. Rajagopal, R. - "Stevia as an alternative sweetener - Promise and Challenges", KnowGenix \& Centre for Management Technology (2009). Disponível em: <www.steviaworldforum.com/steviapaper/ SteviaPosition-P.pdf>. Accesso em: 5 fev. 2011.

3. Crammer, B. \& Ikan, R. - "Progress in the Chemistry and Properties of Rebaudiosides", in: Developments in Sweeteners, v.3, T. H. Grenby (ed.), Elsevier Applied Science, London (1987).

4. Phillips, K. C. - "Stevia: Steps in Developing a New Sweetener", in: Developments in Sweeteners, v.3, T. H. Grenby (ed.), Elsevier Applied Science, London (1987).

5. Takamura, K.; Kawaguchi, M. \& Isono, C. - "Stevioside from Stevia rebaudiana", Japanese Patent No 52047961 (in japanese) (1977).

6. Abou-Arab, A. E., Abou-Arab, A. A. \& Abu-Salem, M. F. - African Journal of Food Science, 4(5), p.269 (2010).

7. Silva, F. V.; Bergamasco, R.; Andrade, C. M. G.; Pinheiro, N.; Machado, N. R. C. F.; Reis M. H.; Araújo, Á. A. \& Rezende, S. - Int. J. Chem. React. Eng., 5, p.A40 (2007). http://dx.doi.org/10.2202/1542-6580.1443

8. Reis, M. H. M.; Silva, F. V.; Andrade, C. M. G.; Rezende, S. L.; Maciel, M. R. W. \& Bergamasco, R. - J. Food Proc. Eng., 32, p.338 (2009).

9. Fernandes, L. M.; Pereira, N. C.; Mendes, E. S.; Lima, C. O. M. \& Costa, S. C. - Acta Sci., 23, p.1369 (2001).

10. Bunhak, E. J.; Mendes, E. S.; Pereira, N. C. \& Costa, S. C. - Acta Sci., 24, p.1643 (2002).

11. Mantovaneli, I. C. C.; Ferretti, E. C.; Simões, M. R. \& Silva, C. F. - Braz. J. Chem. Eng., 21, p.449 (2004). http://dx.doi.org/10.1590/ S0104-66322004000300009

12. Mahl, C. R. A.; Oliveira, S. P. D.; Simões, M. R. \& Silva, C. F. - Adsorption Sci. \& Tech., 28, p.189 (2010).

13. Tharanathan, R. N. \& Kittur, F. S. - Crit. Rev. Food Sci. \& Nutr., 43, p.61 (2003). http://dx.doi.org/10.1080/10408690390826455

14. Synowiecki, J. \& Al-Khatteb, N. A. A. - Crit. Rev. Food Sci. Nutr., 43, p.145 (2003). http://dx.doi.org/10.1080/10408690390826473

15. Guibal, E.; Vooren, V. M.; Dempsey, B. A. \& Roussy, J. - Sep. Sci. Tech., 41, p.2487 (2006). 
16. Zeng, D.; Wu, J. \& Kennedy, J. F. - Carbohydr. Polym., 71, p.135 (2008). http://dx.doi.org/10.1016/j.carbpol.2007.07.039

17. Renault, F.; Sancey, B.; Charles, J.; Morin-Crini, N.; Badot, P-M; Winterton, P. \& Crini, G. - Chem. Eng. J., 155, p.775 (2009). http:// dx.doi.org/10.1016/j.cej.2009.09.023

18. Godos, I.; Guzman, H. O.; Soto, R.; García-Encina, P. A.; Becares, E.; Muñoz, R. \& Vargas, V. A. - Biores. Tech., 102, p.923 (2011). http:// dx.doi.org/10.1016/j.biortech.2010.09.036

19. Wang, J.-P.; Chen, Y.-Z.; Ge, X.-W \& Yu H.-Q. - Coll. \& Surf. A: Phys. Eng. Asp., 302, p.204 (2007)
20. Fuh, W. S. \& Chiang, B. H. - J. Food Sci., 55, p.1454 (1990). http:// dx.doi.org/10.1111/j.1365-2621.1990.tb03956.x

21. Costa, S. C.; Nishiyama, P.; Kusumoto, I. T.; Vieira, L. G. E. \& Alavarez, M. - Arq. Biol. Tecn., 34, p.425 (1991)

22. Haaland, P. D. - "Experimental design in biotechnology", Marcel Dekker Inc, New York (1989).

23. Renault, F.; Sancey, B.; Badot, P.-M. \& Crini, G. - Eur. Pol. J., 45, p.1337 (2009). http://dx.doi.org/10.1016/j.eurpolymj.2008.12.027

Enviado: $24 / 03 / 11$

Reenviado: 02/01/12 Aceito: 05/01/12 